\title{
Histopathology and Immunohistochemistry Assessments of Acute Experimental Infection by Brucella melitensis in Bucks
}

\author{
Nurrul Shaqinah Nasruddin1, Mazlina Mazlan1, Mohd Zamri Saad*, Hazilawati Hamzah², \\ Jasni Sabri ${ }^{3}$ \\ ${ }^{1}$ Research Centre for Ruminant Diseases, Faculty of Veterinary Medicine, Universiti Putra Malaysia, Selangor, \\ Malaysia \\ ${ }^{2}$ Department of Veterinary Pathology and Microbiology, Faculty of Veterinary Medicine, Universiti Putra \\ Malaysia, Selangor, Malaysia \\ ${ }^{3}$ Faculty of Veterinary Medicine, Universiti Malaysia Kelantan, Kelantan, Malaysia \\ Email: ${ }^{*}$ mzamri@upm.edu.my
}

Received 11 December 2013; revised 10 January 2014; accepted 17 January 2014

Copyright (C) 2014 by authors and Scientific Research Publishing Inc.

This work is licensed under the Creative Commons Attribution International License (CC BY).

http://creativecommons.org/licenses/by/4.0/

CC) (i) Open Access

\begin{abstract}
Background: Brucellosis in male goats is characterized by arthritis, orchitis and epididymitis, which may induce infertility. Nevertheless, these lesions were categorized as chronic while acute lesions had not been described. This study investigates the histopathological and immuno histochemistry reactions in organs of bucks acutely infected by Brucella melitensis. Results: Only testis and prepuce of acutely infected bucks showed significantly severe histological lesions. Other internal organs had mild to moderate lesions. However, positive immunohistochemistry stainings were observed in organs except the bulbourethral gland. There was a significant positive correlation between the distribution of $B$. melitensis and IHC intensity but no significant correlation between the IHC intensity and histopathology lesions. Conclusion: The results indicate that acute brucellosis did not lead to clinical presentation, although $B$. melitensis was well distributed in various organs of bucks.
\end{abstract}

\section{Keywords}

Histopathology, Immunohistochemistry, Brucella melitensis, Bucks

${ }^{*}$ Corresponding author. 


\section{Introduction}

Brucella melitensis is a Gram negative, facultative intracellular, coccobacillus, non-capsulated and non-spore forming bacterial pathogen [1]. In goats, brucellosis causes serious economic impact to goat farmers from abortions and stillbirths [2]. Infections by $B$. melitensis occur through mucous membrane of the nasopharynx as well as through ingestion of contaminated feed [3].

Brucellosis in male goats is characterized by arthritis, orchitis and epididymitis, which may induce infertility at later stage [4]. Clinical signs vary depending on age, reproductive status, immunological state, and route of infection and virulency of the organism [5]. Post mortem lesions include necrotizing orchitis, epididymitis, seminal vasculitis and prostatitis [6]. Nevertheless, the lesions in reported field cases were categorized as chronic while acute lesions had not been described.

Immunohistochemistry (IHC) technique is a sensitive and specific test that detects Brucella antigen [7]. Using this technique, the organism could be detected in the cytoplasm of inflammatory cells, especially the neutrophils and macrophages of fixed tissues [8]. This report describes the histopathology changes in bucks and correlates them with immunohistochemistry reactions in acute experimental $B$. melitensis infection.

\section{Methods}

\subsection{Animals}

Nine clinically healthy adult bucks, weighing between 25 - $30 \mathrm{~kg}$ were selected and divided into three equal groups. They were housed in well ventilated sheds, in which each group was kept in separate pens at stocking rate of $0.2 \mathrm{~m}^{2}$ per animal [9]. Prior to the start of the experiment, all bucks were tested with Rose Bengal Plate Test (Synbiotics, USA) to ensure that they were free of brucellosis [10]. Cut grass was provided at $2 \mathrm{~kg} / \mathrm{animal} /$ day with supplemented feed at the rate of 300 g/animal/day. Drinking water was available Ad libitum.

\subsection{Inoculum}

A field strain of B. melitensis isolated earlier from an outbreak of caprine brucellosis [10] was sub-cultured onto Brucella agar (BBL ${ }^{\mathrm{TM}}$, USA) and incubated at $37^{\circ} \mathrm{C}$ for $96 \mathrm{~h}$. Pure colonies were harvested into phosphate buffer saline (PBS) before an inoculum was prepared at the concentration of $10^{9} \mathrm{cell} / \mathrm{ml}$ using the McFarland Standard [11].

\subsection{Experimental Design}

At the start of the experiment, goats of Groups 1 and 2 were exposed intra-conjunctival with $50 \mu \mathrm{L}$ of the inoculum into each eye [12]. Goats of Group 3 were similarly exposed with $50 \mu \mathrm{L}$ of sterile normal saline. All goats were observed for clinical signs following infection before goats of Group 1 were killed on day-7 post-infection (PI). Goats of Groups 2 and 3 were killed on day-14 PI. The conjunctiva, synovial membrane of knee joint, lymph nodes (submandibular, prescapular and supramammary), liver, spleen, testis, prepuce, epididymis, bulbourethral and seminal vesicle glands were collected and fixed in $10 \%$ buffered formalin. The experimental procedure was according to the Guidelines of the Animal Care and Use Committee, Universiti Putra Malaysia (IACUC NO: 09R50).

\subsection{Histopathology}

Fixed tissues were processed in tissue processor (Leica TP 1020, Germany) followed by embedding in paraffin wax, sectioned at $4 \mu \mathrm{m}$ (Leica Jung Multicut 2045, Germany) and stained with Harris' haematoxylin and eosin (HE). Histopathological assessments were made using Nikon Eclipse 50i, Japan and the specific criteria, which included necrosis, atrophy, haemorrhage, congestion, infiltration of inflammatory cells and hyperplasia were scored as follow; 0: none, 1: 30\% affected, 2: 30\% - 60\% affected and 3: $>60 \%$ affected [8].

\subsection{Immunohistochemistry}

Brucella melitensis antigens were demonstrated by using the avidin-biotin-peroxidase complex immune histochemistry staining method. The slides were dried at $60^{\circ} \mathrm{C}$ for $15 \mathrm{~min}$ before deparaffinised in xylene for 10 min and rehydrated through a series of graded alcohol. Heat mediated antigen retrieval was used by immersing the 
slides in citrate buffer solution and heated in microwave oven for $10 \mathrm{~min}$ at low temperature. Blocking with 3\% hydrogen peroxidase for $5 \mathrm{~min}$ at room temperature inactivated the indigenous peroxidase. The sections were washed with PBS and blocked with 5\% bovine serum albumin (Gibco, USA) for $15 \mathrm{~min}$ at $37^{\circ} \mathrm{C}$, followed with overnight incubation at $4^{\circ} \mathrm{C}$ with primary antibody prepared in rabbit $(1: 100)$. The sections were then incubated with secondary antibody (IgG goat anti rabbit) (Abcam, UK) for $1 \mathrm{~h}$ at $37^{\circ} \mathrm{C}(1: 500)$. The slides were rinsed and washed with PBS before diaminobenzidine DAB (Dako, USA) was used as chromogen for 15 sec. Harris's haematoxylin stain was used as counter stain. The degree of IHC staining was scored as 0: no staining, 1: light brown staining, 2: brown staining, 3: golden brown staining, while the degree of distribution was graded as 0 : none, 1: focal, 2: multifocal and 3: diffuse [9].

\subsection{Statistical Analysis}

Histopathology and immunohistochemistry scores were subjected to Mann-Whitney and Pearson's Correlation test using the predictive analysis software 20 (PASW version 20; SPSS Inc., Chicago, IL, USA) by comparing the lesion severity between groups. The significance value was at $\mathrm{p}<0.05$.

\section{Results}

No clinical signs were observed in all groups during the 14-day experimental period. Similarly, no gross lesion was observed.

Histologically, neutrophilic orchitis was observed in all goats of Groups 1 and 2, and there was no significant ( $>0.05$ ) difference in the severity of orchitis between the two acutely infected groups (Table 1). All convoluted seminiferous tubules in the affected areas of the testis were severely necrotic with karyolitic and vacuolated spermatogonia, some were found detached from the basement. Some Sertoli cells were moderately atrophied and the loosening of tunica vasculosa caused the tubules to appear wrinkled. Neutrophilic and lymphocytic cell infiltrations were observed within the interstitial tissues (Figure 1). Immunohistochemistry revealed that the antigen was distributed both focal and multifocal, particularly within the cytoplasm of spermatogonia, Sertoli cells and the surrounding neutrophils and necrotic debris (Figure 1). Following IHC, the epithelial cells of epididymis were stained with light to intense brown while distribution of the antigen was significantly $(\mathrm{p}<0.05)$ higher in goats of Group 2 (Table 2).

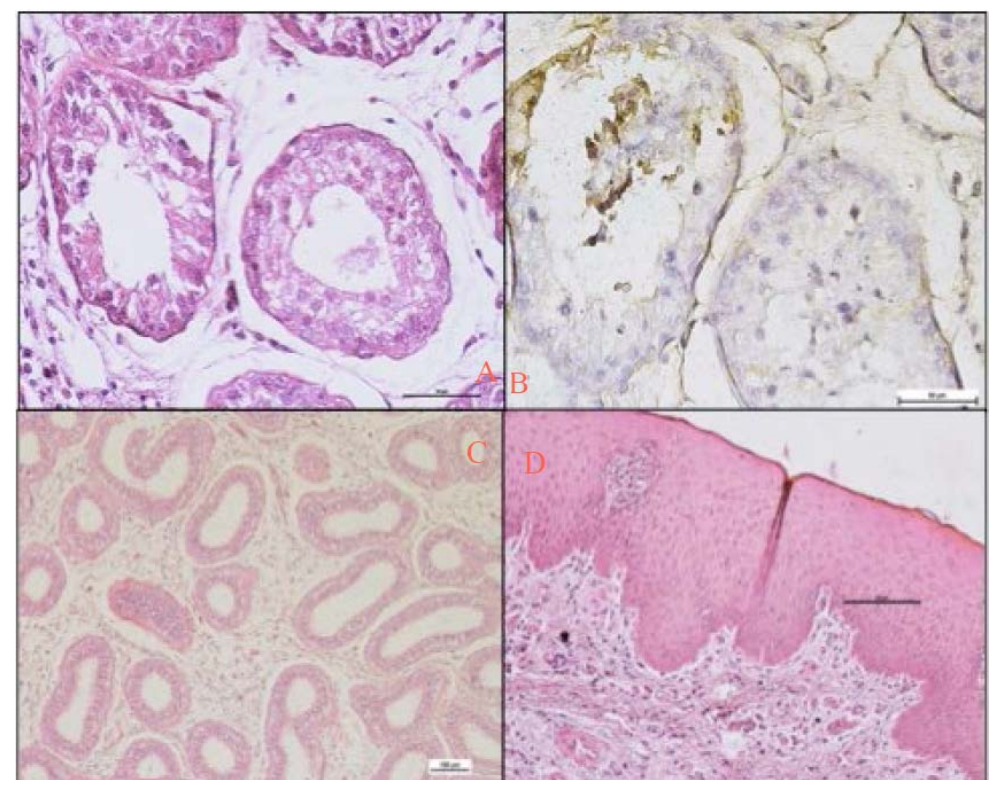

Figure 1. (A) Necrotic orchitis with diffuse lymphocyte and neutrophilic infiltrations. HE, $\times 400$; (B) Brucella melitensis antigens (brown) with the necrotic tissues. IHC, $\times 400$; (C) Epididymitis with severe neutrophilic infiltration. HE, $\times 100$; (D) Neutrophilic posthitis with formation of mucosal-associated lymphoid tissue (MALT) in the mucosal epithelium. HE, $\times 200$. 
Table 1. Results for histopathological lesion reading in mean \pm SD with different letter represent significant differences $(p$ $<0.05)$. Results of every organ were compared accordingly to the type of lesion for the two different treatments. ICI: Inflammatory cell infiltration.

\begin{tabular}{|c|c|c|c|}
\hline ORGAN & LESION & 7 DAY & 14 DAY \\
\hline \multirow{5}{*}{ Testis } & Necrosis & $2.67 \pm 0.24$ & $2.67 \pm 0.24$ \\
\hline & Atrophy & $1.60 \pm 1.12$ & $2.20 \pm 0.68$ \\
\hline & Congestion & $0.80 \pm 0.86$ & $0.73 \pm 0.80$ \\
\hline & ICI & $2.47 \pm 0.52$ & $2.27 \pm 0.59$ \\
\hline & Average & $1.88 \pm 1.07$ & $1.97 \pm 0.97$ \\
\hline \multirow{2}{*}{ Epididymis } & Inflm. cell infiltration & $1.33 \pm 0.90$ & $1.07 \pm 0.70$ \\
\hline & Average & $1.33 \pm 0.90$ & $1.07 \pm 0.70$ \\
\hline \multirow{4}{*}{ Prepuce } & Necrosis & $0.40 \pm 0.51$ & $0.93 \pm 0.59^{\mathrm{a}}$ \\
\hline & Congestion & $1.27 \pm 0.88$ & $0.93 \pm 0.96$ \\
\hline & Inflm. cell infiltration & $1.73 \pm 1.10$ & $1.80 \pm 0.68$ \\
\hline & Average & $1.13 \pm 1.01$ & $1.27 \pm 0.84$ \\
\hline \multirow{7}{*}{ Bulbourethral } & Necrosis & - & - \\
\hline & Atrophy & - & - \\
\hline & Haemorrhage & - & - \\
\hline & Congestion & - & - \\
\hline & Inflam. cell infiltration & - & - \\
\hline & Hyperplasia & - & - \\
\hline & Average & - & - \\
\hline \multirow{4}{*}{ Seminal Vesicle } & Congestion & $0.67 \pm 0.49$ & $0.80 \pm 0.77$ \\
\hline & Inflam. cell infiltration & $0.73 \pm 0.70$ & $1.07 \pm 0.80$ \\
\hline & Hyperplasia & - & - \\
\hline & Average & $0.70 \pm 0.59$ & $0.93 \pm 0.78$ \\
\hline \multirow{4}{*}{ Submandibular LN } & Congestion & $0.87 \pm 0.92$ & $1.27 \pm 0.70$ \\
\hline & Inflam. cell infiltration & $0.60 \pm 0.51$ & $0.93 \pm 0.59$ \\
\hline & Hyperplasia & $0.27 \pm 0.46$ & $1.13 \pm 0.83^{\mathrm{a}}$ \\
\hline & Average & $0.58 \pm 0.59$ & $1.11 \pm 0.71^{\mathrm{a}}$ \\
\hline \multirow{5}{*}{ Pre-scapular LN } & Haemorrhage & $0.53 \pm 0.52$ & $0.67 \pm 0.82$ \\
\hline & Congestion & $0.73 \pm 0.70$ & $0.67 \pm 0.72$ \\
\hline & Inflam. cell infiltration & $0.53 \pm 0.52$ & $0.73 \pm 0.80$ \\
\hline & Hyperplasia & $0.53 \pm 0.64$ & $1.20 \pm 1.01^{\mathrm{a}}$ \\
\hline & Average & $0.58 \pm 0.69$ & $0.82 \pm 0.85$ \\
\hline \multirow{3}{*}{ Supra-mammary LN } & Inflam. cell infiltration & $0.47 \pm 0.52$ & $0.80 \pm 0.68$ \\
\hline & Hyperplasia & - & - \\
\hline & Average & $0.47 \pm 0.52$ & $0.80 \pm 0.68$ \\
\hline \multirow{4}{*}{ Liver } & Congestion & $0.00 \pm 0.00$ & $2.60 \pm 0.51^{\mathrm{a}}$ \\
\hline & Inflam. cell infiltration & $0.00 \pm 0.00$ & $1.00 \pm 0.53^{\mathrm{a}}$ \\
\hline & Hyperplasia & - & - \\
\hline & Average & $0.00 \pm 0.00$ & $2.07 \pm 0.91^{\mathrm{a}}$ \\
\hline
\end{tabular}




\begin{tabular}{|c|c|c|c|}
\hline Continued & & & \\
\hline \multirow{7}{*}{ Spleen } & Necrosis & - & - \\
\hline & Atrophy & - & - \\
\hline & Haemorrhage & - & - \\
\hline & Congestion & - & - \\
\hline & Inflam. cell infiltration & $0.60 \pm 0.51$ & $0.93 \pm 0.59$ \\
\hline & Hyperplasia & $0.87 \pm 0.92$ & $1.27 \pm 0.70$ \\
\hline & Average & $0.73 \pm 0.74$ & $1.10 \pm 0.66^{\mathrm{a}}$ \\
\hline \multirow{4}{*}{ Synovial Membrane } & Congestion & $0.27 \pm 0.46$ & $0.87 \pm 0.74^{\mathrm{a}}$ \\
\hline & Inflam. cell infiltration & $0.67 \pm 0.72$ & $1.13 \pm 0.35^{\mathrm{a}}$ \\
\hline & Hyperplasia & - & - \\
\hline & Average & $0.47 \pm 0.62$ & $1.00 \pm 0.58^{\mathrm{a}}$ \\
\hline \multirow{4}{*}{ Conjunctiva } & Congestion & $0.40 \pm 0.51$ & $0.67 \pm 0.62$ \\
\hline & Inflam. cell infiltration & $0.93 \pm 0.80$ & $1.47 \pm 0.64^{\mathrm{a}}$ \\
\hline & Hyperplasia & - & - \\
\hline & Average & $0.67 \pm 0.71$ & $1.07 \pm 0.74$ \\
\hline
\end{tabular}

a indicates significant $(\mathrm{p}<0.05)$ difference.

Table 2. Results for distribution of Brucella meitensis in organs in mean \pm SD with different letter represent significant differences $(p<0.05)$. Results of every organ were compared accordingly to the distribution of antigens for two different treatments. LN: Lymph nodes.

\begin{tabular}{ccc}
\hline ORGAN & Group 1 & Group 2 \\
\hline Testis & $1.60 \pm 0.99$ & $1.87 \pm 0.99$ \\
Epididymis & $2.00 \pm 0.76$ & $2.40 \pm 0.51$ \\
Prepuce & $2.20 \pm 0.56$ & $1.60 \pm 1.06$ \\
Bulbourethral & - & - \\
Seminal vesicle & $2.80 \pm 0.41$ & $2.60 \pm 0.51$ \\
Submandibular Lymph Node & $2.47 \pm 0.92$ & $1.87 \pm 0.99$ \\
Pre-scapular LN & $1.87 \pm 1.13$ & $1.60 \pm 1.12$ \\
Supra-mammary LN & $1.93 \pm 1.10$ & $1.67 \pm 1.05$ \\
Liver & $0.60 \pm 0.51$ & $2.0 \pm 0.65^{\mathrm{a}}$ \\
Spleen & $1.67 \pm 0.62$ & $1.80 \pm 0.56$ \\
Synovial membrane & $1.60 \pm 1.24$ & $2.0 \pm 1.13$ \\
Conjunctiva & $2.00 \pm 0.76$ & $2.40 \pm 0.51$ \\
\hline
\end{tabular}

a indicates significant $(\mathrm{p}<0.05)$ difference.

Both infected groups demonstrated posthitis although Group 2 revealed significantly $(\mathrm{p}<0.05)$ higher lesion score. The stratified squamous epithelium showed vacuolation with infiltration of numerous inflammatory cells, particularly the neutrophils, macrophages and lymphocytes that extended into the connective tissue and muscular layers. There were occasional mucosal associated lymphoid tissues (MALT) in the prepuce epithelial and interstitial layers of the infected goats of Groups 1 and 2 (Figure 1). The IHC staining showed similar distribution but significantly $(p<0.05)$ higher intensity scoring in Group 1 . The positive reactions were found mainly in the stratified squamous epithelium and the endothelium of small vessels within the connective tissues (Figure 2).

Neither seminal vesiculitis nor bulbourethritis was observed in all groups. However, the seminal vesicle revealed golden brown reactions within cytoplasm of the columnar epithelium and the muscularis layer (Figure 2). 


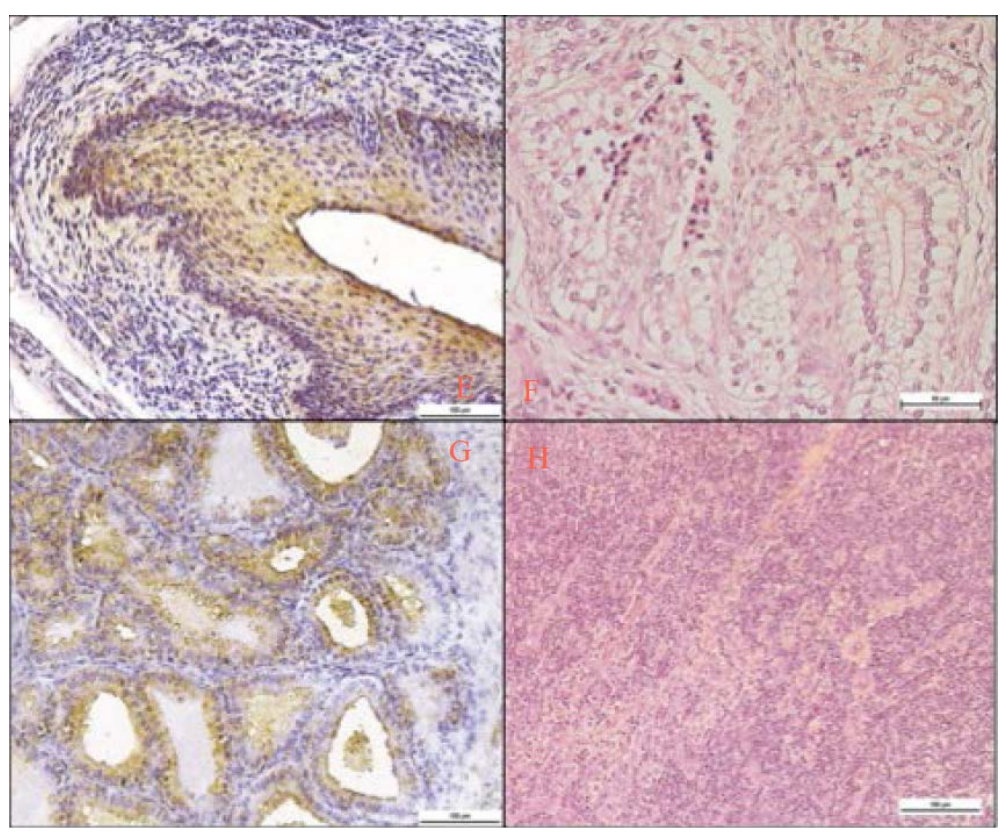

Figure 2. (E) B. melitensis antigens demonstrated with strong immuno staining in the mucosal epithelium of prepuce. IHC, $\times 200$; (F) Seminar vesiculitis with mild lympho-neutrophilic Interstititial infiltration. HE, $\times 400$; $(G)$ The antigens are present in the cytoplasm of columnar epithelium as well as in the lumen. IHC, ×200; (H) Hyperplastic lymphadenitis was observed in supra mammary lymph nodes. HE, $\times 200$.

Lymphadenitis was observed in both infected groups and hyperplasia was frequently observed in the submandibular and pre-scapular lymph nodes (Figure 2). Generally, the IHC results for lymph nodes were either multifocal or diffuse with brown to golden brown. The reactions were observed in the cytoplasm of inflammatory cells, especially the lymphocytes and neutrophils.

Hepatitis was significantly $(\mathrm{p}<0.05)$ severe in Group 2 . The hepatocytes were swollen with loss of architecture while some of hepatocytes showed karyolysis. Infiltrations of neutrophils were observed surrounding the central vein (Figure 3). Most IHC stains were observed involving hepatocytes near the central vein and within the cytoplasm of neutrophils. Most IHC stains were observed involving hepatocytes near the central vein and within the cytoplasm of neutrophils.

Goats of Group 2 showed significantly $(\mathrm{p}<0.05)$ severe splenitis with focal to multifocal antigen distribution in moderate intensity (Figure 3). Moreover, the stifle joints of infected goats showed synovitis with infiltrations of few neutrophils. The blood vessels in the affected areas were congested. The antigen can be observed in the squamous epithelium and within the cytoplasm of infiltrating neutrophils (Figure 3).

Moderate conjunctivitis was observed in both infected groups. There were infiltrations of macrophages and neutrophils within the laminapropria that extended into the muscle layer (Figure 4). Early formation of MALT in the stratified squamous epithelial layer was observed in both infected groups. Significantly $(p<0.05)$ intense golden brown staining was observed at tip of the epithelial layers of Group 2 (Table 3). Similarly, the secreting glands and blood vessels were also stained positive (Figure 4).

There was a highly significant positive correlation between the antigen distribution and IHC intensity $(\mathrm{r}=$ 0.971; $\mathrm{p}<0.05)$ in goats of Group 1. Lesser significant positive correlation was calculated $(r=0.595, p<0.05)$ for goats of Group 2. On the other hand, there was insignificant positive correlation between histology and IHC scorings $(r=0.315 . \mathrm{p}>0.05)$ in goats of Group 1 and insignificant negative correlation in Group $2(r=-0.127$, $\mathrm{p}>0.05)$.

\section{Discussion}

The absence of clinical signs and macroscopic lesions is in disagreement to those reported earlier [13], probably 


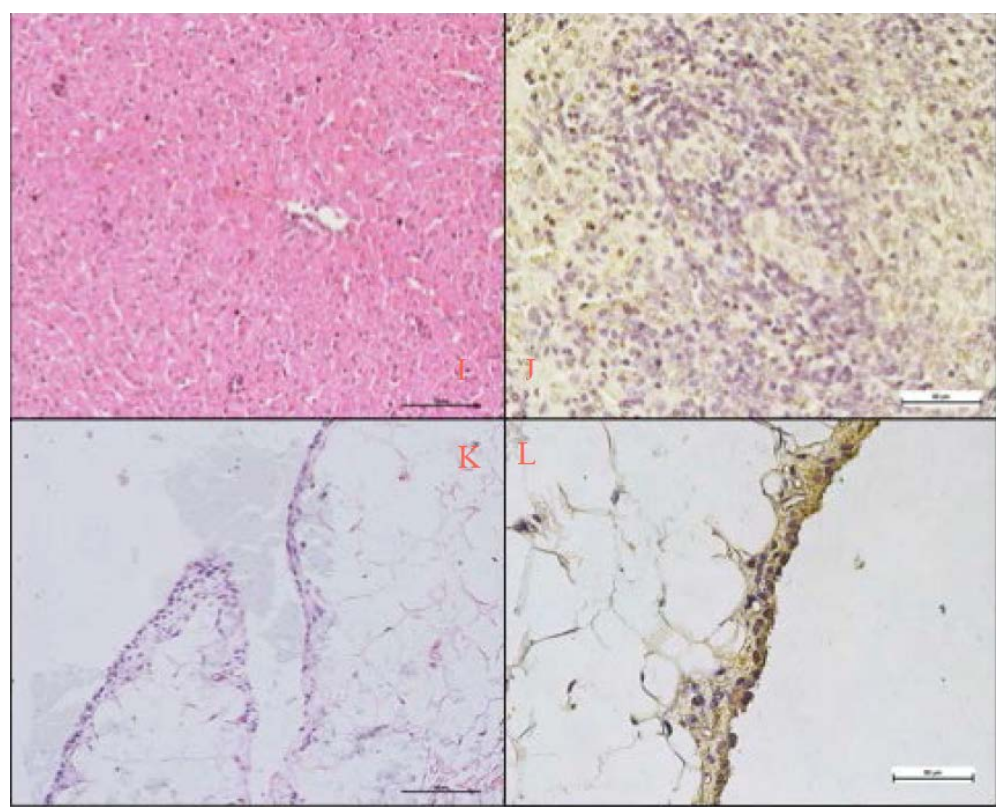

Figure 3. (I) Necrotic hepatitis with aggregation of neutrophils (arrow). HE, $\times 200$; (J) The B. melitensis Antigens were demonstrated in the cypoplasm of inflammatory cells indicated in black circle. IHC, $\times 400$; (K) Mild synovitis of stifle joint. HE, $\times 200$; (L) The synovial membrance demonstrated very intense golden brown immuno staining at the epithelium and in the inflammatory cells. IHC, $\times 400$.

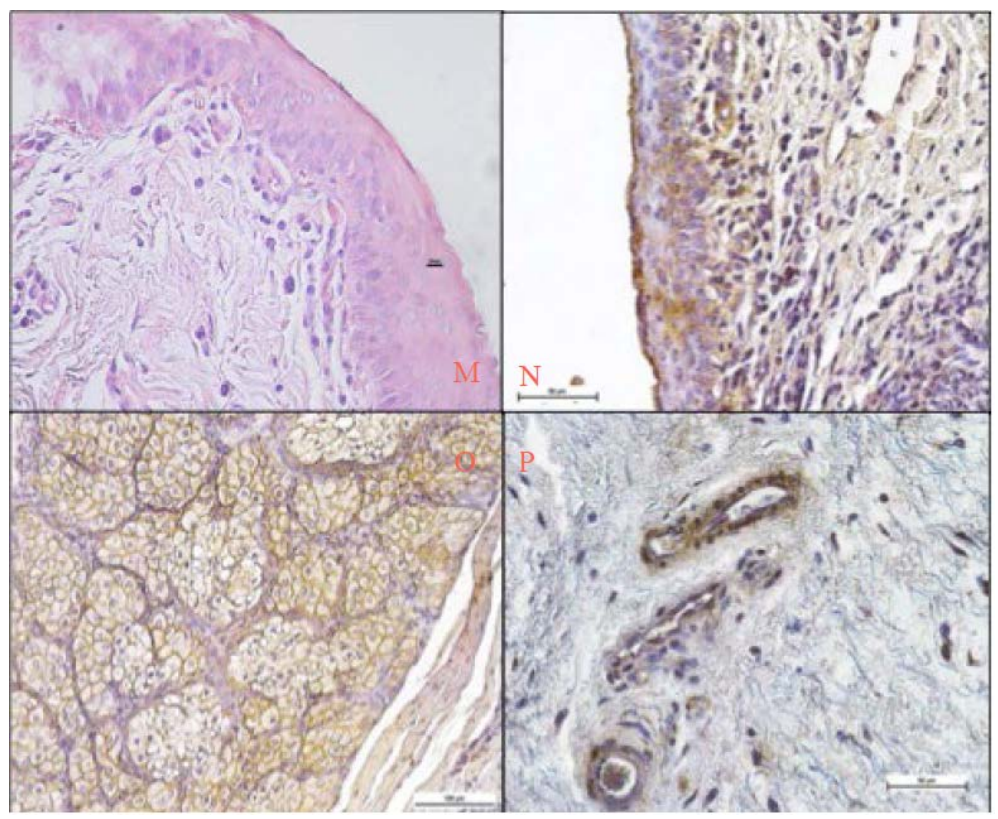

Figure 4. (M) Conjunctivitis with moderate interstitial accumulation of inflammatory cells. HE, $\times 400$; $(\mathrm{N}) \mathrm{B}$. melitensis antigens were detected in the interstitial area as well as in the cytoplasm of stratified epithelium cells. IHV, $\times 400$; (O) Lacrimal gland were stained with intense immuno labeling stain. IHC, ×400; (P) Endothelial cells demonstrated positive IP staining. IHC, $\times 400$.

due to the difference in exposure time and route of infection.

The necrotic neutrophilic orchitis had also been reported in acute infection by Arcanobacterium pyogenes in 
Table 3. Results for IHC staining intensity of Brucella meitensis in organs in mean \pm SD with different letter represent significant differences $(\mathrm{p}<0.05)$. Results of every organ were compared accordingly to the intensity of immuno staining for two different treatments. LN: Lymph nodes.

\begin{tabular}{ccc}
\hline ORGAN & Group 1 & Group 2 \\
Testis & $1.73 \pm 1.03$ & $1.80 \pm 0.94$ \\
Epididymis & $1.87 \pm 0.64$ & $2.73 \pm 0.46^{\mathrm{a}}$ \\
Prepuce & $2.27 \pm 0.70^{\mathrm{a}}$ & $1.40 \pm 1.06$ \\
Bulbourethral & - & - \\
Seminal vesicle & $2.47 \pm 0.52$ & $1.87 \pm 0.92$ \\
Submandibular Lymph Node & $2.20 \pm 0.77 \pm 0.70$ \\
Pre-scapular LN & $1.73 \pm 1.16$ & $2.13 \pm 1.19$ \\
Supra-mammary LN & $1.73 \pm 1.16$ & $1.80 \pm 1.21$ \\
Liver & $0.93 \pm 0.80$ & $1.93 \pm 0.80^{\mathrm{a}}$ \\
Spleen & $1.67 \pm 0.82$ & $1.87 \pm 0.74$ \\
Synovial membrane & $1.53 \pm 1.19$ & $1.73 \pm 0.96$ \\
Conjunctiva & $1.87 \pm 0.64$ & $2.73 \pm 0.46^{\mathrm{a}}$
\end{tabular}

a indicates significant $(\mathrm{p}<0.05)$ difference.

fection in bucks [14]. The severe orchitis may lead to reproductive problems if the lesions remain [4]. The large numbers of $B$. melitensis antigens were observed intracellular within the macrophages and neutrophils, effectively enhanced the survival by avoiding fusion with lysosomes [15]. Similarly, the antigen was found extracellularly within the necrotic debris causing massive destruction to the seminiferous tubules [7]. The mild inflammation of the epididymis was similar to the previous study [13], although the lesions in this study were concentrated in the tail of epididymis.

The formation of MALT in the prepuce epithelium cells was in response to the presence of $B$. melitensis in the lumen, therefore, it is postulated that $B$. melitensis was capable of traveling through the blood vessels from conjunctiva to the prepuce before being shed into the preputial lumen and becoming a source of infection through copulation.

Although both seminal vesicles and bulbourethral glands showed no histopathological changes, the IHC staining proved the presence of the antigen in the seminal vesicles which was described in naturally infected bucks, whereby more localization of $B$. melitensis was observed in the seminal vesicles than the epididymis [16].

Mild to moderate histopathology lesions were observed in the liver, probably due to the short duration of exposure. The few neutrophilic infiltrations with brown immunohistochemistry staining around the central vein of liver indicated the haematogenous route of spread of $B$. melitensis [17]. In human brucellosis, hepatitis is a common lesion [18] to be found but not in animals [17].

It was reported that bucks infected with $B$. melitensis may show arthritis [19], which is in agreement with the findings of this study, particularly of Group 2. Furthermore, a study showed that naturally infected brucellosis in camels demonstrated pyoarthritis and hygroma [20]. Immunostaining proved the evidence of multifocal distribution of antigen in the synovial membranes which suit the suggestion on the use of joint hygroma fluid as one of the clinical samples for isolation of the organism [21].

\section{Conclusion}

This study revealed no correlation between the histology and immunohistochemistry results, which contradicted an earlier report [8]. This was likely due to the acuteness of the infection that led to insufficient time for the body to significantly react to the infection. On the other hand, the distribution was positively correlated with the intensity, indicating that the more antigens were distributed, the higher concentration of antigen and the more 
intense immunohistochemistry staining.

\section{Authors' Contributions}

NNS: contributed substantially to the design of the study and sampling, drafting of the manuscript. MM: involved in sampling and analysis of results and contributed in drafting of the manuscript. MZS: contributed in conception and design of the study, supervising the protocol and critical review of the manuscript. HH: substantially important in the experimental design, coordination, interpretation of results and critical review of the manuscript. JS: played an important role in the review of the manuscript.

\section{Acknowledgements and Funding}

The authors acknowledged the help of the staff of Histopathology Laboratory and Post-Mortem Unit, Faculty of Veterinary Medicine, Universiti Putra Malaysia. The study was financially supported by the FRGS research grant of the Ministry of Higher Education Malaysia.

\section{References}

[1] Seleem, M.N., Boyle, S.M. and Sriranganathan, N. (2010) Brucellosis: A Re-Emerging Zoonosis. Veterinary Microbiology, 140, 392-398. http://dx.doi.org/10.1016/j.vetmic.2009.06.021

[2] Gupta, V.K., Rout, P.K. and Vihan, V.S. (2007) Induction of Immune Response in Mice with a DNA Vaccine Encoding Outer Membrane Protein (omp31) of Brucella melitensis 16M. Research in Veterinary Science, 82, 305-313. http://dx.doi.org/10.1016/j.rvsc.2006.07.014

[3] Abdullah, F.F.J., Adamu, L., Hazirah, N., Osman, A. Y., Mansor, R., Harun, A.W., Zamri-Saad, M., Omar, A.R. and Saharee, A.Z. (2013) Clinical and Reproductive Pathological Changes Associated with Brucella melitensis and Its Lipopolysaccharides in Female Mice via Oral Inoculation. American Journal of Animal and Veterinary Science, 8, 104-111. http://dx.doi.org/10.3844/ajavsp.2013.104.111

[4] Neta, A.V.C., Mol, J.P.S., Xavier, M.N., Paixão, T.A., Lage, A.P. and Santos, R.L. (2010) Pathogenesis of Bovine Brucellosis. Veterinary Journal, 184, 146-155. http://dx.doi.org/10.1016/j.tvjl.2009.04.010

[5] Adams, L.G. (2002) The Pathology of Brucellosis Reflects the Outcome of the Battle between the Host Genome and the Brucella Genome. Veterinary Microbiology, 90, 553-561. http://dx.doi.org/10.1016/S0378-1135(02)00235-3

[6] Xavier, M.N., Paixão, T.A., Hartigh, A.B., Tsolis, R.M. and Santos, R.L. (2010) Pathogenesis of Brucella spp. Veterinary Science Journal, 4, 109-118.

[7] Ilhan, F. and Yener, Z. (2008) Immunohistochemical Detection of Brucella melitensis Antigens in Cases of Naturally Occurring Abortions in Sheep. Journal of Veterinary Diagnostic Investigation, 20, 803-806. http://dx.doi.org/10.1177/104063870802000616

[8] Xavier, M.N., Paixa o, T.A., Poester, F.P., Lage, A.P. and Santos, R.L. (2009) Pathological, Immunohistochemical and Bacteriological Study of Tissues and Milk of Cows and Fetuses Experimentally Infected with Brucella abortus. Journal of Comparative Pathology, 140, 149-157. http://dx.doi.org/10.1016/j.jcpa.2008.10.004

[9] Emikpe, B.O., Tanko, P.N., Onilude, O.M. and Sabri, M.Y. (2013) The Influence of Dexamethasone Treatment and Successive Road Transport Stress on the Occurrence of Caprine Pneumonia in a Hot Humid Tropical Environment. Veterinary World, 6, 497-501.

[10] Plumeriastuti, H. and Zamri-Saad, M. (2012) Detection of Brucella melitensis in Seropositive Goats. Online Journal of Veterinary Research, 16, 1-7.

[11] McFarland, J. (1907) An Instrument for Estimating the Number of Bacteria in Suspensions Used for Calculating the Opsonic Index and Vaccines. Journal of American Medical Information Association, 14, 1176-1178. http://dx.doi.org/10.1001/jama.1907.25320140022001f

[12] Suraud, V., Jacques, I., Olivier, M. and Guilloteau, L.A. (2008) Acute Infection by Conjunctival Route with Brucella melitensis Induces IgG $^{+}$Cells and IFN- $\gamma$ Producing Cells in Peripheral and Mucosal Lymph Nodes in Sheep. Microbes and Infection, 10, 1370-1378. http://dx.doi.org/10.1016/j.micinf.2008.08.003

[13] Carvalho Júnior, C.A., Moustacas, V.S., Xavier, M.N., Costa, E.A., Costa, L.F., Silva, T.M.A., Paixão, T.A., Borges, A.M., Gouveia, A.M.G. and Santos, R.L. (2012) Andrological, Pathologic, Morphometric, and Ultrasonographic Findings in Bucks Experimentally Infected with Brucella ovis. Small Ruminant Research, 102, 213-222. http://dx.doi.org/10.1016/j.smallrumres.2011.08.004

[14] Gouletsou, P.G., Fthenakis, G.C., Cripps, P.J., Papaioannou, N., Lainas, T., Psalla, D. and Amiridis, G.S. (2004) Experimentally Induced Orchitis Associated with Arcanobacterium pyogenes: Clinical, Ultrasonographic, Seminological 
and Pathological Features. Theriogenology, 62, 1307-1328.

[15] Celli, J. (2006) Surviving Inside a Macrophage: The Many Ways of Brucella. Research in Microbiology, 157, 93-98.

[16] Jansen, B.C. (1980) The Pathology of Bacterial Infection of the Genitalia in Bucks. Onderstepoort Journal of Veterinary Research, 47, 263-267.

[17] Mense, M.G., Richard, H., Borschel, R.H., Wilhelmsen, C.L., Louise Pitt, M.L. and Hoover, D.L. (2004) Pathologic Changes Associated with Brucellosis Experimentally Induced by Aerosol Exposure in Rhesus Macaques (Macaca mulatta). American Journal of Veterinary Research, 65, 644-652. http://dx.doi.org/10.2460/ajvr.2004.65.644

[18] Colmenero Jde, D., Queipo-Ortuño, M.I., Maria Reguera, J., Angel Suarez-Muñoz, M., Martín-Carballino, S. and Morata, P. (2002) Chronic Hepatosplenic Abscesses in Brucellosis. Clinico-Therapeutic Features and Molecular Diagnostic Approach. Diagostic Microbiology and Infectious Diseases, 42, 159-167.

[19] OIE (2009) Caprine and Ovine Brucellosis (Excluding Brucella ovis). Manual of Diagnostic Tests and Vaccines for Terrestrial Animals, http://www.oie.int/fileadmin/Home/eng/Health_standards/tahm/2.07.02_caprine_Ovine_bruc.pdf (accessed 07.10.13).

[20] Gwida, M., El-Gohary, A., Melzer, F., Khan, I., Rösler, U. and Neubauer, H. (2012) Brucellosis in Camels. Research in Veterinary Science, 92, 351-355. http://dx.doi.org/10.1016/j.rvsc.2011.05.002

[21] Poester, F.P., Nielsen, K., Samartino, L.E. and Yu, W.L. (2010) Diagnosis of Brucellosis. Open Veterinary Science Journal, 4, 46-60. 